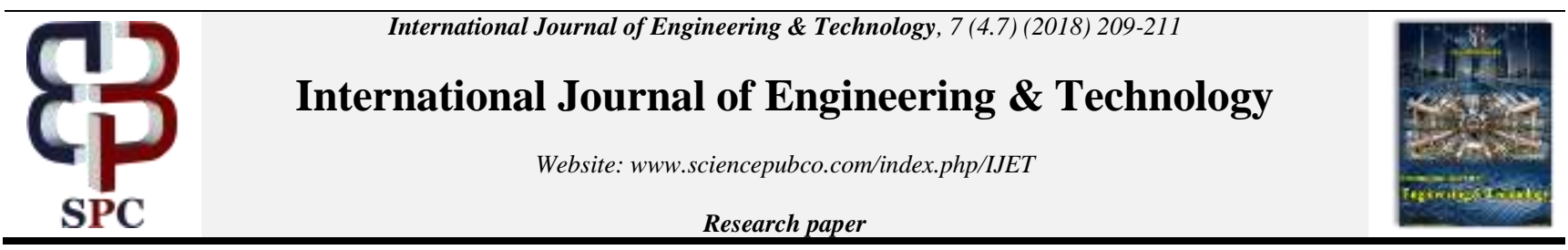

\title{
Mathematical Modelling and Optimization of Risk Analysis in Emergencies
}

\author{
Korneev A. M., Salfetnikov M.V., Glazkova Y. A., Lavrukhina T. V., Abdullakh L.S. \\ Lipetsk state technical University, Russia \\ *Corresponding author E-mail: d.48@ rambler.ru
}

\begin{abstract}
In current work methods of intellectual support of acceptance of administrative decisions on risks of occurrence of extreme situations are considered. The relationship between human activity, the emergence emergencies and the state of the environment is systemic. An important role is given to the analysis of the causes of incidents and emergencies of technogenic nature. Emergencies refers to complex multi-level systems, so a systematic approach is needed to model them. When choosing models of complex systems, factors related to the natural properties of the constituent elements and subsystems, and the patterns of the functioning of the system as a whole are investigated. Various methods of mathematical modeling are used to determine the risks of emergencies. The problem of optimization of decisionmaking algorithms in the event of emergencies is solved.
\end{abstract}

Keywords: optimization, economic indicators, GA, algorithms, risk analysis, Mathematical Model.

\section{Introduction}

Emergency situations cause significant human and material losses. An important task is to analyze the trends of change and the scale of the consequences of natural and technogenic emergency situations. To correct the situation, it is necessary to use modern methods of research and management of the state of industrial and ecological systems.

The relationship between human activity, the emergence of emergencies and the state of the environment is systemic.

It is necessary to investigate systemic connections and patterns of functioning of a complex of objects on which emergencies are possible.

Emergencies refers to complex multi-level systems, so a systematic approach is needed to model them.

When choosing models of complex systems, factors related to the natural properties of the constituent elements and subsystems, and the patterns of the functioning of the system as a whole are investigated.

The process of preventing, localizing and eliminating emergencies (especially for long-term, medium-term, operational forecasts of the threat of emergencies) should be based on complete and reliable information, and have sufficient time reserve for making a decision on emergency assistance to the population in the emergency area.

To determine the risks of emergencies and identify priority areas for making decisions on the risks of emergencies, various methods of mathematical modeling are used.

The adoption of managerial decisions on the risks of emergencies has the character of a heuristic iterative process. The peculiarity of the developed method of calculations - systematic review of multidimensional areas formed on the basis of natural factors.

At the first stage, the original boundaries of the natural factors studied are determined. The initial ones in this case are the bound- aries forming the region of the actual natural subspace of the factors.

\section{Mathematical Model}

The choice of the subspace of factors (factors of emergence of emergency situations) begins with the construction of a grid - the partition of the parameter space $\Xi$ into a set of subspaces (alternatives) $\begin{aligned} \Xi_{\mu} & \\ & \Xi=\underset{\mu}{\bigcup} \Xi_{\mu}\end{aligned}$

A grid is a set of subspaces. The most uniform view of an ndimensional cube is provided by a cubic lattice. The number $\mu$ subspaces in the analysis process can vary depending on the degree of detail of the alphabets used in the random variables studied. The total number of subspaces can reach this:

$$
\boldsymbol{\mu}=\prod_{k=1}^{K} \prod_{m_{k}=1}^{M_{k}} J_{m_{k}}
$$

Each factor of occurrence of emergency situations varies within certain limits: $x_{i}{ }^{\prime} \leq x_{i} \leq x_{i}{ }^{\prime \prime}$. To analyze the risks of emergencies, the initial boundaries forming the subspace $\Xi_{j}$. 


$$
\begin{aligned}
& X^{\prime u}=\left(x_{1 m_{1}}^{\prime u}, \ldots x_{k m_{k}}^{\prime u}, \ldots x_{K m_{K}}^{\prime u}\right) \\
& X^{\prime \prime} u=\left(x_{1 m_{1}}^{u}, \ldots x_{k m_{k}}^{u}, \ldots x_{K m_{K}}^{\prime u}\right)
\end{aligned}
$$

where $k$ - kind of factors of occurrence of extreme situations, $j_{k}-$ $j$-th random variable $k$-th kind of factors.

For each random variable, the minimum and maximum values for the sample are determined $x_{k m_{k}}^{{ }^{\prime}}, x_{k m_{k}}^{{ }^{\prime \prime}}$. Then this range of variation of the input quantity is divided into a number of constituent alphabets: $b_{k m_{k} 1}, b_{k m_{k} 2}, \ldots, b_{k m_{k} j_{m_{k}}}, \ldots, b_{k m_{k} J_{m_{k}}}$ where $k$ - kind of factors of emergencies, $m_{k}$ - random variable of the $k$-th kind of factors, $j_{m_{k}}=1, \ldots, J_{m_{k} \text { - numbers of the }}$ components of the alphabet of a given value). Each selected component of the alphabet

$$
\begin{aligned}
& b_{k m_{k} j_{m_{k}}}=\left(x_{k m_{k} j_{m_{k}}}^{{ }^{\prime} \mu}, x_{k m_{k} j_{m_{k}}}^{\prime \prime}\right) \\
& \text { Where } x_{k m_{k} j_{m_{k}}}^{{ }^{\prime} n}, x_{k m_{k} j_{m_{k}} \text { - boundaries of the }}^{{ }^{n}{ }^{\prime n}} j_{m_{k} \text { se- }}
\end{aligned}
$$
lected component of the alphabet, $H$ - initial setting:

$$
x_{k m_{k} j_{m_{k}}}^{\prime \prime \prime}=x_{k m_{k}}^{{ }^{\prime}}+\left(j_{m_{k}}-1\right)\left(x_{k m_{k}}^{{ }^{6}}-x_{k m_{k}}^{{ }^{\prime}}\right) / J_{m_{k}}
$$

$x_{k m_{k} j_{m_{k}}{ }^{H}}=x_{k m_{k}}^{{ }^{6}}+j_{m_{k}}\left(x_{k m_{k}}^{{ }^{6}}-x_{k m_{k}}^{\prime 6}\right) / J_{m_{k}}$

Numerical values $j_{m_{k}}$ are chosen in such a way that any halfinterval contains a sufficient number of points (experiments) obtained in the study of values of a random variable ${ }^{\mathrm{km}_{k}}$. Variants of combinations of alphabets of the investigated quantities:

$$
\xi_{\beta_{(k)}}=b_{(k) 1 j_{1}}, \ldots, b_{(k) m_{k} j_{m_{k}}}, \ldots, b_{(k) M_{k} j_{M_{k}}}
$$

The level of properties (the combination of emergency risks) is in most cases a random value or, at least, not strictly deterministic. Therefore, it is possible for each mode to set the probabilities of obtaining a certain level of properties, i.e. probability of realization of various combinations of risks of emergency situations.

\section{Results and Analysis}

Information for all variants of combinations of alphabets at the frequency of occurrence of a combination $X>0$ entered in the table 1 .

The resulting table is an M-dimensional histogram. Therefore, it is necessary to obtain this table from the input data.

Table 1:. "Non-zero" subspaces of emergency factors

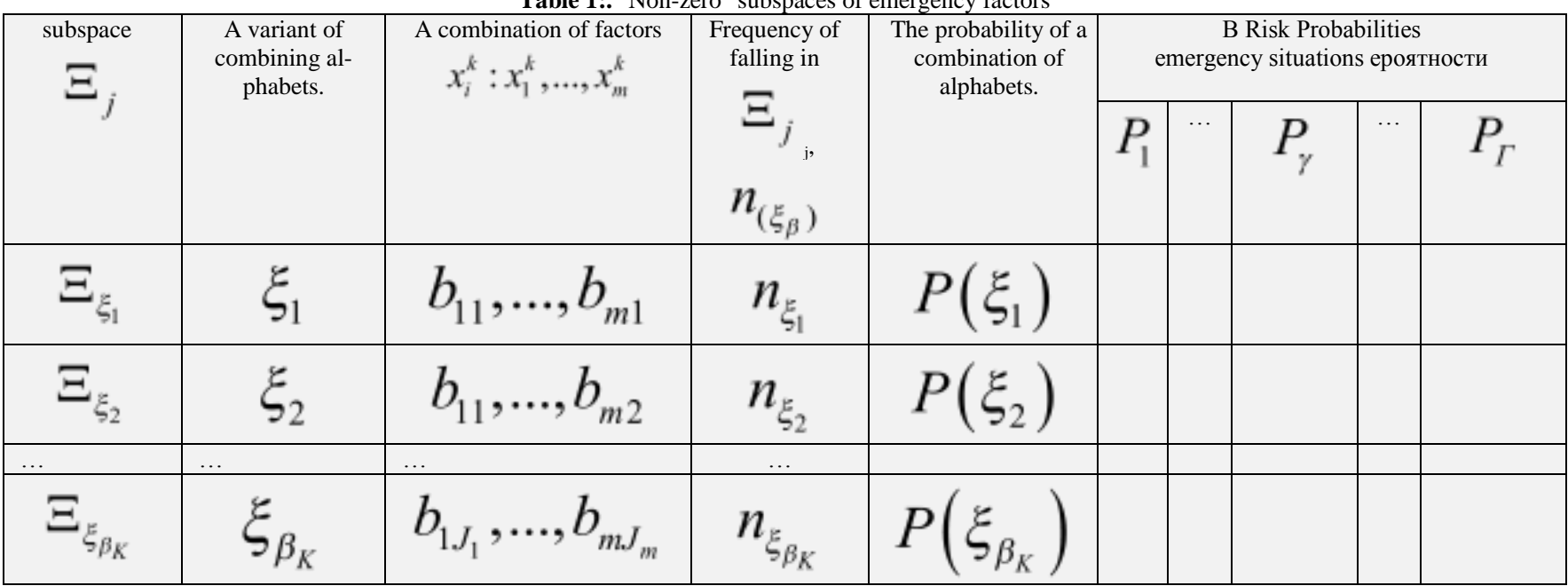
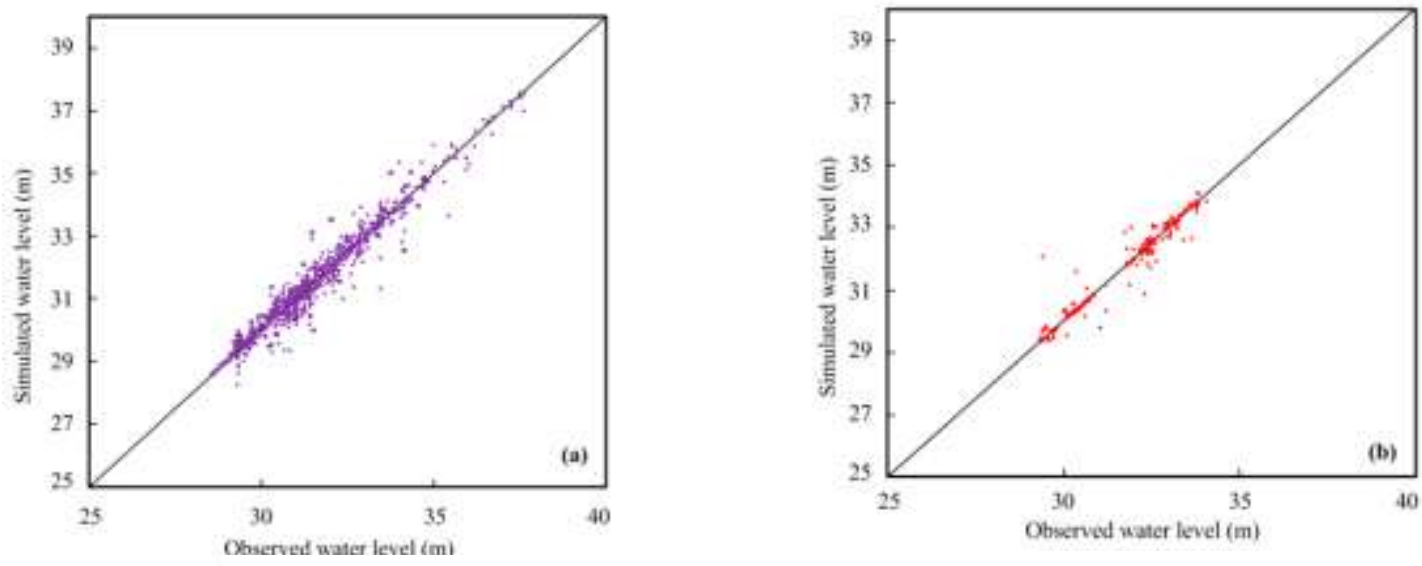

Figure 1: The optimization procedure 
The probability of emergency risks is defined as the ratio of the number of emergencies $n_{\gamma \xi_{\beta_{K}}}$ for the subset under study $\Xi_{j}$ to the total number of realizations of a given subset;

$$
P_{\gamma \xi_{\beta_{K}}}=\frac{n_{\gamma \xi_{\beta_{K}}}}{n_{\xi_{\beta_{K}}}}
$$

Mathematical model of dependence of probability of emergency ${ }_{\text {situations }} P_{\gamma}$ from the factors of occurrence of emergency situa$\mathcal{X}_{\text {tions }}{ } \mathrm{mm}_{k}$, is represented as:

$$
P_{\gamma}=\sum_{k=1}^{K} \sum_{m_{k}=1}^{M_{k}} \alpha_{k m_{k}} f_{k m_{k}}\left(x_{k m_{k}}\right)
$$

where $f_{k m_{k}}$ - the chosen optimal functions of the connection of the factors causing emergencies and the likelihood of emergency situations, is the kind of factors causing emergencies, mk is the random variable of the k-th kind of factors, $\alpha_{k m m_{k}}-$ the model coefficients obtained..

The number of rows of the sample to be created for each subspace is equal to the number of hits in the given subset $\left(n_{\xi_{\beta_{K}}}\right)$. In this case, the coordinates of the inputs are the real values of all points

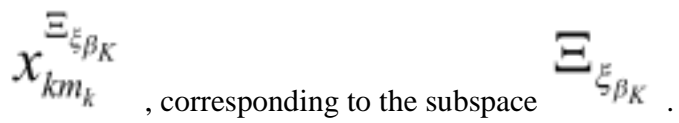

\section{Conclusion}

The article considers methods of intellectual support for making managerial decisions on the risks of emergencies. An important role is played by the analysis of the causes of accidents and emergencies of anthropogenic nature. Emergencies refers to complex multi-level systems, so a systematic approach is needed to model them. When choosing models of complex systems, factors related to the natural properties of the constituent elements and subsystems, and the patterns of the functioning of the system as a whole are investigated. Various methods of mathematical modeling are used to determine the risks of emergencies. The problem of optimization of decision-making algorithms in the event of emergencies is solved. A subspace of emergency factors is formed and the probability of emergency risks is monitored for each subspace.

\section{References}

[1] A.M. Korneev, L.S.Abdullakh, T.A. Smetannikova, criterion for assessing the effectiveness of functioning of complex systems based on the use the amount of information, International Journal of Applied Engineering Research. 2015. T. 10. № 11. C. 27961-27980

[2] Abdullah L.S, Korneev A.M, Smetannikova T.A. Structural cellhierarchical identification of complex spatially distributed production systems // Proceedings of the 3rd International Academic Conference. 2013, St. Louis, Missouri, USA. C.75-79.

[3] S.L. Blyumin, A.M. Shmyrin, O.A. Shmyrina. Bilinear Neighborhood Systems. Monograph. Lipetsk, Lipetsk State Technical University Publishers, 2006. 131 p, (in Russian).

[4] Korneev, A. M. Methods for the identification of cross-cutting technology production of steel products: monograph / Lipetsk state pedagogical University. - Lipetsk: LGU, 2009. - 286 p.
[5] Korneev, A. M. cellular Structural-hierarchical modeling of complex prostranstvenno-distributed systems. - News of higher educational institutions of the Central Chernozem region, 2011, No. 1, pp. 62-66.

[6] Analysis of economic indicators of complex production processes/ Korneev A.M., Abdullah L.S. // International Journal of Engineering \& Technology Vol. 7(3.5), 2018, pp. 7-9.

[7] Identification of complex production systems with using iterative networks / Korneev A.M., Abdullakh L.S., Sukhanov A.B., Antar, S.D., Al-jonid Kh.M// International Journal of Engineering \& Technology Vol. 7(3.5), 2018, pp. 37-39.

[8] Stratonovich, R. L. Theory of information. - Moscow: Sov. radio, 1975. - 424 p. Korneev, A. M. the Use of information criteria connection input and output variables for the selection of optimal technologies - Izvestiya vuzov. Ferrous metallurgy, 2004, No. 9, p. 48 52.

[9] A. M. Shmyrin, I. A. Sedykh, Identification and Control Algorithms of Functioning for Neighborhood Systems Based on Petri Nets, Automation and Remote Control, 2010, Volume 71, Issue 6, p. 1265-1274.

[10] Kuznetsov, L. A., A. Korneev, M. Automated system of selecting the optimal technology for the production of steel. - Izvestiya vuzov. Ferrous metallurgy, 1994, No. 5, pp. 45-48. 